TRANSACTIONS OF THE

AMERICAN MATHEMATICAL SOCIETY

Volume 350, Number 10, October 1998, Pages 4221-4233

S 0002-9947(98)02012-1

\title{
COMPUTATIONS IN GENERIC REPRESENTATION THEORY: MAPS FROM SYMMETRIC POWERS TO COMPOSITE FUNCTORS
}

\author{
NICHOLAS J. KUHN
}

\begin{abstract}
If $\mathbf{F}_{q}$ is the finite field of order $q$ and characteristic $p$, let $\mathcal{F}(q)$ be the category whose objects are functors from finite dimensional $\mathbf{F}_{q}$-vector spaces to $\mathbf{F}_{q}$-vector spaces, and with morphisms the natural transformations between such functors. Important families of objects in $\mathcal{F}(q)$ include the families $S_{n}, S^{n}, \Lambda^{n}, \bar{S}^{n}$, and $c T^{n}$, with $c \in \mathbf{F}_{q}\left[\Sigma_{n}\right]$, defined by $S_{n}(V)=$ $\left(V^{\otimes n}\right)^{\Sigma_{n}}, S^{n}(V)=V^{\otimes n} / \Sigma_{n}, \Lambda^{n}(V)=n^{t h}$ exterior power of $V, \bar{S}^{*}(V)=$ $S^{*}(V) /\left(p^{t h}\right.$ powers $)$, and $c T^{n}(V)=c\left(V^{\otimes n}\right)$.

Fixing $F$, we discuss the problem of computing $\operatorname{Hom}_{\mathcal{F}(q)}\left(S_{m}, F \circ G\right)$, for all $m$, given knowledge of $\operatorname{Hom}_{\mathcal{F}(q)}\left(S_{m}, G\right)$ for all $m$. When $q=p$, we get a complete answer for any functor $F$ chosen from the families listed above.

Our techniques involve Steenrod algebra technology, and, indeed, our most striking example, when $F=S^{n}$, arose in recent work on the homology of iterated loopspaces.
\end{abstract}

\section{INTRODUCTION}

As in our series of papers [K:I, K:II, K:III], if $\mathbf{F}_{q}$ is the finite field of order $q$ and characteristic $p$, let $\mathcal{F}(q)$ be the abelian category with objects the functors

$$
F \text { : finite dimensional } \mathbf{F}_{q} \text {-vector spaces } \longrightarrow \mathbf{F}_{q} \text {-vector spaces, }
$$

and with morphisms the natural transformations. We like to view an object $F \in$ $\mathcal{F}(q)$ as a 'generic representation' of the general linear groups over $\mathbf{F}_{q}$, as $F(V)$ becomes an $\mathbf{F}_{q}[G L(V)]$-module for all $\mathbf{F}_{q}$-vector spaces $V$. The tight relationship, explored in [K:II], between $\mathcal{F}(q)$ and the categories of $\mathbf{F}_{q}\left[G L_{n}\left(\mathbf{F}_{q}\right)\right]$-modules, for all $n$, makes the study of $\mathcal{F}(q)$ of great representation theoretic interest.

We call $F \in \mathcal{F}(q)$ simple if it has no nontrivial subobjects, finite if it has a finite composition series with simple subquotients ${ }^{1}$, and locally finite if it is the union of its finite subobjects. The full subcategory consisting of the locally finite functors is denoted by $\mathcal{F}_{\omega}(q)$.

Let us introduce some examples of interest. $I$ is the 'inclusion' functor: $I(V)=$ $V$. The families $T^{n}, S_{n}, S^{n}$, and $\bar{S}^{n}$ are defined, for $n \geq 0$, by $T^{n}(V)=V^{\otimes n}, S_{n}(V)$ $=\left(V^{\otimes n}\right)^{\Sigma_{n}}, S^{n}(V)=V^{\otimes n} / \Sigma_{n}$, and $\bar{S}^{*}(V)=S^{*}(V) /\left(p^{t h}\right.$ powers $)$. All of these are finite, and the $\bar{S}^{n}$ are simple, as are the exterior powers $\Lambda^{n}$. Note that $T^{n}$ admits

Received by the editors September 11, 1996 and, in revised form, January 3, 1997.

1991 Mathematics Subject Classification. Primary 20G05; Secondary 55S10, 55S12.

Partially supported by the N.S.F. and the C.N.R.S.

${ }^{1}$ The finite functors are also characterized as the functors taking finite dimensional values, and that are polynomial in the sense of Eilenberg-Mac Lane $[\mathrm{EM}]$ (or anyone else, e.g. [MacD]). See [K:I, Appendix A]. 
an $\mathbf{F}_{q}\left[\Sigma_{n}\right]$ action; thus, for any $c \in \mathbf{F}_{q}\left[\Sigma_{n}\right]$, we have a functor $c T^{n}$. For a fixed $\mathbf{F}_{q}$-vector space $W$, let $J_{W}(V)=\mathbf{F}_{q}^{\operatorname{Hom}(V, W)}$. The $J_{W}$ are locally finite injectives satisfying $\operatorname{Hom}_{\mathcal{F}(q)}\left(F, J_{W}\right)=F(W)^{*}$. (See [K:I, K:II] for further discussion of these points.)

More complex functors can be built out of these by using the operations $F \otimes G$ and $F \circ G$. The point of this paper is to discuss the following problem.

Problem. For a fixed $F$, compute $\operatorname{Hom}_{\mathcal{F}(q)}\left(S_{*}, F \circ G\right)$, in terms of $\operatorname{Hom}_{\mathcal{F}(q)}\left(S_{*}, G\right)$.

A number of remarks are in order here.

\section{Remarks 1.1.}

(1) Firstly, $\operatorname{Hom}_{\mathcal{F}(q)}\left(S_{*}, G\right)$ means the graded vector space $\bigoplus_{n=0}^{\infty} \operatorname{Hom}_{\mathcal{F}(q)}\left(S_{n}, G\right)$. This has extra structure, to be described shortly.

(2) $F \circ G$ is only obviously defined if $G$ takes finite dimensional values. For a general $G \in \mathcal{F}(q)$, we define $F \circ G \in \mathcal{F}(q)$ by letting $(F \circ G)(V)$ be the colimit of $F(W)$, with the colimit ranging over finite dimensional $W \subset G(V)$.

(3) There are natural isomorphisms $[\mathrm{K}: \mathrm{I}]^{2}$ :

$$
\operatorname{Hom}_{\mathcal{F}(q)}\left(S_{*}, F\right) \otimes \operatorname{Hom}_{\mathcal{F}(q)}\left(S_{*}, G\right) \simeq \operatorname{Hom}_{\mathcal{F}(q)}\left(S_{*}, F \otimes G\right)
$$

The results in this paper, together with this 'Kunneth formula', suffice to give routine algorithms for computing $\operatorname{Hom}_{\mathcal{F}(q)}\left(S_{*}, G\right)$, where $G$ is any functor built up from the examples defined above, using tensor products and compositions, under the restriction that $q$ is prime.

(4) There is a duality functor $D: \mathcal{F}(q)^{o p} \rightarrow \mathcal{F}(q)$ defined by $D F(V)=F\left(V^{*}\right)^{*}$, under which $S_{n}$ and $S^{n}$ are dual ${ }^{3}$. If $G$ takes finite dimensional values, then there is an isomorphism $\operatorname{Hom}_{\mathcal{F}(q)}\left(F \circ G, S^{*}\right) \simeq \operatorname{Hom}_{\mathcal{F}(q)}\left(S_{*}, D F \circ D G\right)$. Thus our results give information about maps from composite functors to symmetric coinvariants. (The reader is warned, however, that the dual of a locally finite functor need not be locally finite.)

(5) Our methods are based on modern versions of algebraic topologists' 'Steenrod algebra technology'. We are dubious that our results can be attained without using these.

To state our main results, we need to explain how the Steenrod algebra enters. Let $\mathcal{S}^{*}$ be the full subcategory of $\mathcal{F}(q)$ with objects $S^{n}$, and then let $\mathcal{U}(q)=$ $\operatorname{Rep}\left(\mathcal{S}^{*}\right)$, the category of additive functors from $\mathcal{S}^{*}$ to $\mathbf{F}_{q}$ vector spaces. Thus $M \in$ $\mathcal{U}(q)$ is a graded vector space equipped with suitably compatible maps $a: M_{m} \rightarrow$ $M_{n}$ for all $a: S^{m} \rightarrow S^{n}$. An observation in [K:I] is that all such $a: S^{m} \rightarrow S^{n}$ arise though the action of Steenrod operations on $S^{*}(V)$, and that $\mathcal{U}(q)$ is precisely the category of unstable modules over the (Hopf) algebra $A(q)$ of Steenrod reduced $q^{t h}$ powers ${ }^{4}$.

For any $M \in \mathcal{U}(q)$, the $q^{\text {th }}$ power maps $\Phi: S^{n} \rightarrow S^{q n}$ induce a map $\Phi: M \rightarrow M$, which multiplies degree by $q$. We let $\mathcal{U}_{\Phi}(q)$ denote the category of graded $\mathbf{F}_{q}$-vector spaces having such a self map, and we let $\mathcal{U}_{\emptyset}(q)$ be the category of graded $\mathbf{F}_{q}$-vector spaces. Then there are forgetful functors $\mathcal{U}(q) \rightarrow \mathcal{U}_{\Phi}(q) \rightarrow \mathcal{U}_{\emptyset}(q)$.

\footnotetext{
${ }^{2}$ This extends to $\operatorname{Ext}_{\mathcal{F}(q)}^{*}$. See [K:III].

${ }^{3}$ Less obviously, simple functors are self dual. See [K:II, §7].

${ }^{4} \mathrm{~A}$ module $M$ is unstable if it satisfies $a x=0$ whenever the excess of $a$ is greater than the degree of $x$. For example, when $q=2$, this means that $S q^{i} x=0$ if $i>|x|$.
} 
Let $M_{G}=\operatorname{Hom}_{\mathcal{F}(q)}\left(S_{*}, G\right)$. The 'extra structure' on $M_{G}$ arises from noting that $M_{G} \in \mathcal{U}(q)$. This is perhaps a good place to recall the basic computation : $M_{I}=$ $\left\langle x_{k} \mid k=0,1, \ldots\right\rangle$, where $x_{k}$ has degree $q^{k}$ and corresponds to $\Phi^{k}: I=S^{1} \rightarrow S^{q^{k}}$ (see $[\mathrm{K}: \mathrm{I}])$.

Our first observation gives content to the problem posed above.

Theorem 1.2. Given $F \in \mathcal{F}(q)$, there exists a functor $U_{F}: \mathcal{U}(q) \rightarrow \mathcal{U}(q)$ and natural isomorphisms of unstable $\mathcal{A}(q)$-modules, $U_{F}\left(M_{G}\right) \simeq M_{F \circ G}$ for all $G \in$ $\mathcal{F}_{\omega}(q)$.

Our remaining results amount to explicit descriptions of $U_{F}$ 's, for various types of functors $F \in \mathcal{F}(q)$, sometimes with the restriction that $q$ be prime.

All of the functors $\Lambda^{n}, S_{n}, S^{n}, \bar{S}^{n}, c T^{n}$ extend in an obvious way to $\mathcal{U}(q)$, a category with a tensor product.

Theorem 1.3. There are natural isomorphisms in $\mathcal{U}(q), F\left(M_{G}\right) \simeq M_{F \circ G}$, in the following cases:

(1) $F=S_{n}$,

(2) $F=\Lambda^{n}$,

(3) $F=\bar{S}^{n}$,

(4) $F=c T^{n}$, with $c \in \mathbf{F}_{q}\left[\Sigma_{n}\right]$

Corollary 1.4. In these cases, $M_{F \circ G}$ just depends on $M_{G}$ as a graded vector space.

Remarks 1.5 .

(1) Note that we have omitted the family $F=S^{n}$. For good reason: see Theorem 1.9 , below.

(2) Cases (2) and (3) are special cases of (4). However, they have technically simpler proofs.

(3) When $q$ is prime, case (4) includes all the simple functors. In particular, if $F_{\lambda}$ is simple, then $F_{\lambda}\left(M_{I}\right)$ computes the occurrences of $F_{\lambda}$ in the socle of $S^{n}$, for all $n$. This was already noted in [KK, §3], where the nonprime field case was also briefly discussed. See also [FS] for a somewhat different proof when $q=2$, and our discussion in $\S 5$.

Let $\mathcal{K}(q)$ be the category of commutative, unital algebras $K$ in $\mathcal{U}(q)$ (a category with a tensor product) satisfying the 'restriction axiom': $\Phi(x)=x^{q}$ for all $x \in K$.

Let $U: \mathcal{U}(q) \rightarrow \mathcal{K}(q)$ be the free functor, left adjoint to the forgetful functor. Explicitly, $U(M)=S^{*}(M) /\left(\Phi(x)-x^{q}\right)$.

Theorem 1.6. $M_{J_{W} \circ G}$ is naturally an object in $\mathcal{K}(q)$, with algebra structure induced from that on the ring of functions $J_{W}(V)$. If $q$ is prime, there are natural isomorphisms in $\mathcal{K}(q), U\left(M_{G} \otimes W^{*}\right) \simeq M_{J_{W} \circ G}$.

Here $W^{*}$ is to be viewed as an unstable $\mathcal{A}(q)$-module concentrated in degree 0 .

Note that if $\Phi: M \rightarrow M$ is monic, then $U(M)$ is (not naturally) isomorphic to $S^{*}(M / \Phi(M))$ as an algebra, and thus is polynomial. Thus the theorem has the following corollary.

Corollary 1.7. If $q$ is prime, as a graded algebra, $M_{J_{W} \circ G}$ only depends on $M_{G}$ as an object in $\mathcal{U}_{\Phi}(q)$, and is polynomial. The Poincaré series of $M_{J_{W}} \circ G$ only depends on the Poincaré series of $M_{G}$. 
Remarks 1.8.

(1) If $q$ is not a prime, $U\left(M_{G} \otimes W^{*}\right)$ need not equal $M_{J_{W} \circ G}$, though it does when $G=I$.

(2) The functor $U$ has long been in the topology literature (see e.g. [SE]). One learns that, at least at the prime 2, the cohomology of Eilenberg Mac Lane spaces has a representation theoretic description: there are isomorphisms in $\mathcal{K}(2)$,

$$
H^{*}\left(K(W, n) ; \mathbf{F}_{2}\right) \simeq \operatorname{Hom}_{\mathcal{F}(2)}\left(S_{*}, J_{W} \circ S_{n}\right),
$$

natural in both $W$ and $n$.

To describe how $M_{S^{n} \circ G}$ depends on $M_{G}$, we need variants of the categories $\mathcal{U}(q)$ and $\mathcal{K}(q)$. As in $[\mathrm{K}: \mathrm{III}]$, let $\mathcal{U}^{2}(q)$ be $\operatorname{Rep}\left(\mathcal{S}^{*} \otimes \mathcal{S}^{*}\right)$, i.e., the category of bigraded modules over the bigraded (Hopf) algebra $A(q) \otimes A(q)$, unstable in each grading. Note that, given $M, N \in \mathcal{U}(q), M \otimes N$ can be regarded as an object in $\mathcal{U}^{2}(q)$. Given $M \in \mathcal{U}^{2}(q)$, there are natural maps $\Phi_{1}: M_{m, *} \rightarrow M_{q m, *}$ and $\Phi_{2}: M_{*, n} \rightarrow M_{*, q n}$, and we let $\Phi=\Phi_{1} \otimes \Phi_{2}: M_{m, n} \rightarrow M_{q m, q n}$. Let $\mathcal{K}^{2}(q)$ denote the category of commutative, unital algebras $K$ in $\mathcal{U}^{2}(q)$ satisfying the 'restriction axiom': $\Phi(x)=x^{q}$ for all $x \in K$. Let $U_{2}: \mathcal{U}^{2}(q) \rightarrow \mathcal{K}^{2}(q)$ be the free functor, left adjoint to the forgetful functor. Explicitly, $U_{2}(M)=S^{*}(M) /\left(\Phi(x)-x^{q}\right)$.

Theorem 1.9. $M_{S^{*} \circ G}$ is naturally an object in $\mathcal{K}^{2}(q)$, with algebra structure induced from that on $S^{*}(V)$. If $q$ is prime, there are natural isomorphisms in $\mathcal{K}^{2}(q)$, $U_{2}\left(M_{G} \otimes M_{I}\right) \simeq M_{S^{*} \circ G}$.

Corollary 1.10. If $q$ is prime, as a bigraded algebra, $M_{S^{*} \circ G}$ only depends on $M_{G}$ as an object in $\mathcal{U}_{\Phi}(q)$, and is polynomial. The Poincaré series of $M_{S^{n} \circ G}$ only depends on the Poincaré series of $M_{G}$.

Remarks 1.11 .

(1) As in the previous remark, if $q$ is not a prime, $U_{2}\left(M_{G} \otimes M_{I}\right)$ need not equal $M_{S^{*} \circ G}$, though it does when $G=I$ [K:III].

(2) Starting from iterated loopspaces on spheres, the author has recently constructed [K2] a bigraded family of topological spectra $X(n, j)$, such that there are $\mathcal{A}(2)$-module isomorphisms

$$
H^{*}\left(X(n, j) ; \mathbf{F}_{2}\right) \simeq \operatorname{Hom}_{\mathcal{F}(2)}\left(S_{*},\left(\Phi^{-1} S^{j}\right) \circ S_{n}\right)=\Phi_{2}^{-1} \operatorname{Hom}_{\mathcal{F}(2)}\left(S_{*}, S^{j} \circ S_{n}\right) .
$$

Our proof, and then use, of this isomorphism depends on Theorem 1.9, and was the initial motivation for our investigations here.

As remarked above, both Theorem 1.6 and Theorem 1.9 do not hold without modification if $q$ is not prime. Our last result illustrates the added complexity of the nonprime field case.

As in [K:II, §5], if $q=p^{s}$ with $s>1$, let $G_{\xi}$ denote the functor $G$ twisted by the Frobenius. Noting that $G_{\xi}=I_{\xi} \circ G$, we see that $G \longmapsto G_{\xi}$ is a composition operator which iterated $s$ times is the identity. In $\S 5$, we will define a functor $U_{\xi}: \mathcal{U}(q) \rightarrow \mathcal{U}(q)$. As graded vector spaces,

$$
U_{\xi}(M)_{n}=\bigcap_{i \geq 1} \operatorname{Ker}\left\{Q^{i}: M_{p n} \rightarrow M_{p n+q^{i}-1}\right\}
$$

where $Q^{i} \in \mathcal{A}(q)$ is the $q$-analogue of the operation $Q^{i} \in \mathcal{A}(p)$ studied by Adams and Wilkerson in $[\mathrm{AW}]$.

Theorem 1.12. There are natural isomorphisms in $\mathcal{U}(q), U_{\xi}\left(M_{G}\right) \simeq M_{G_{\xi-1}}$. 
Thus it appears that, unlike all of our previous examples, $M_{G_{\xi}}$ can only be computed if one knows a lot about the $\mathcal{A}(q)$-module stucture on $M_{G}$.

In $\S 2$ we recall needed results about $\mathcal{F}(q)$ and $\mathcal{U}(q)$. In particular, central to our arguments are the Embedding and Vanishing Theorems of [K:I, K:III], and some of their consequences. Theorem 1.2 then becomes obvious, and a general strategy for explicit computation emerges. Using this, Theorem 1.3 is proved in $\S 3$, and Theorems 1.6, 1.9 in $\S 4$. Theorem 1.12 is proved more directly in $\S 5$.

\section{Prerequisites about $\mathcal{U}(q)$ and $\mathcal{F}(q)$}

Recalling that $\mathcal{U}(q)=\operatorname{Rep}\left(\mathcal{S}^{*}\right)$, very formally, there are adjoint functors

$$
r: \mathcal{F}(q) \rightarrow \mathcal{U}(q),
$$

and

$$
l: \mathcal{U}(q) \rightarrow \mathcal{F}(q),
$$

with $r(F)=\operatorname{Hom}_{\mathcal{F}(q)}\left(S_{*}, F\right)=M_{F}$, and $l$ left adjoint to $r$. Since $r$ is a right adjoint, it is left exact, and commutes with limits. Furthermore, the finiteness of the family $S_{n}$ implies that $r$ commutes with filtered colimits.

In [K:I, §7], it is observed that both the existence of a tensor product in $\mathcal{U}(q)$, and the Kunneth formula of the introduction, are consequences of the two natural isomorphisms:

$$
\begin{aligned}
S^{*}(V) \otimes S^{*}(W) & \simeq S^{*}(V \oplus W), \\
J_{V} \otimes J_{W} & \simeq J_{V \oplus W} .
\end{aligned}
$$

The map of the Kunneth isomorphism, $M_{F} \otimes M_{G} \rightarrow M_{F \otimes G}$, is explicitly defined as follows: given $\alpha: S_{m} \rightarrow F$ and $\beta: S_{n} \rightarrow G, \alpha \otimes \beta$ is sent to the composite

$$
S_{m+n} \stackrel{\Psi}{\longrightarrow} S_{m} \otimes S_{n} \stackrel{\alpha \otimes \beta}{\longrightarrow} F \otimes G .
$$

Here $\Psi: S_{*}(V) \rightarrow S_{*}(V) \otimes S_{*}(V)$ is dual to the multiplication on $S^{*}(V)$.

Papers [K:I, K:III] featured two fundamental theorems about the family $S^{n}$ (and, via duality, the family $S_{n}$ ). The first of these is the following 'Embedding Theorem'.

Theorem 2.1. [K:I] Every finite functor $F \in \mathcal{F}(q)$ embeds in a sum of the form $\bigoplus_{i} S^{n_{i}}$. Equivalently, the family $S_{n}, n \geq 0$, generates $\mathcal{F}_{\omega}(q)$.

Via an appropriate version of the Gabriel-Popescu Theorem [P] (a categorical theorem about one-sided Morita equivalence), this theorem is equivalent to the following corollary.

Corollary 2.2. [K:I] $l: \mathcal{U}(q) \rightarrow \mathcal{F}(q)$ is exact, and the natural map $l(r(F)) \rightarrow F$ is an isomorphism for all $F \in \mathcal{F}_{\omega}(q)$.

The Kunneth formula above asserts that $r$ preserves tensor products. With this and the last corollary, one can deduce

Corollary 2.3. [K:I] $l: \mathcal{U}(q) \rightarrow \mathcal{F}(q)$ preserves tensor products.

(The original proofs of these last corollaries, in the prime field case, are due to Lannes [L], and Henn-Lannes-Schwartz [HLS]. They work heavily in the category $\mathcal{U}(q)$.) 
Let $\Phi^{-1} S^{n}$ denote the locally finite functor

$$
\Phi^{-1} S^{n}=\operatorname{colim}\left\{S^{n} \stackrel{\Phi}{\longrightarrow} S^{q n} \stackrel{\Phi}{\longrightarrow} S^{q^{2} n} \rightarrow \ldots\right\} .
$$

The 'Vanishing Theorem' reads

Theorem 2.4. [K:III] For all $n, \Phi^{-1} S^{n}$ is injective in $\mathcal{F}_{\omega}(q)$.

This again is equivalent to a corollary about $\mathcal{U}(q)$. Call $M \in \mathcal{U}(q)$ nilpotent if $\operatorname{colim}\{M \stackrel{\Phi}{\rightarrow} M \stackrel{\Phi}{\rightarrow} M \stackrel{\Phi}{\longrightarrow} \ldots\}=0^{5}$.

Corollary 2.5. [K:III] $l(M) \simeq 0$ if and only if $M$ is nilpotent.

(The original Steenrod algebra proof of this appeared in [LS].)

Let $\mathcal{N} i l(q) \subset \mathcal{U}(q)$ be the full subcategory of nilpotent modules. As a consequence of Corollary 2.2 and Corollary 2.5, $l$ and $r$ induce an equivalence of abelian categories

$$
\mathcal{U}(q) / \mathcal{N} i l(q) \simeq \mathcal{F}_{\omega}(q)
$$

and $M \rightarrow r(l(M))$ can be thought of as 'localization away from $\mathcal{N} i l(q)$ '. Thus, following standard terminology $[\mathrm{G}]$, we call $M \in \mathcal{U}(q)$ nilclosed if it is isomorphic to a module of the form $r(F)$.

Theorem 1.2 is an immediate consequence of Corollary 2.2.

Proof of Theorem 1.2. Let $U_{F}: \mathcal{U}(q) \rightarrow \mathcal{U}(q)$ be defined by $U_{F}(M)=r(F \circ l(M))$. Then, for all $G \in \mathcal{F}_{\omega}(q), U_{F}\left(M_{G}\right)=r(F \circ l(r(G))) \simeq r(F \circ G)=M_{F \circ G}$.

We remark that a functor $U_{F}: \mathcal{U}(q) \rightarrow \mathcal{U}(q)$ is not uniquely determined by the requirement that $U_{F}\left(M_{G}\right) \simeq M_{F \circ G}$. (The functor defined in this last proof is just the terminal example.) In our most interesting examples, those occurring in Theorem 1.6 and Theorem 1.9, our strategy for finding explicit $U_{F}$ 's will be based on the following consequence of the above.

Theorem 2.6. Suppose a functor $U_{F}: \mathcal{U}(q) \rightarrow \mathcal{U}(q)$ satisfies the two conditions:

(1) $U_{F}$ preserves nilclosed modules.

(2) For all $M \in \mathcal{U}(q), l\left(U_{F}(M)\right) \simeq F \circ l(M)$.

Then, for all $G \in \mathcal{F}_{\omega}(q), U_{F}\left(M_{G}\right) \simeq M_{F \circ G}$.

The point of this theorem is that condition (2) is often easy to verify, due to the good properties of $l$.

We end this section by recalling from [K1] the $q$-analogue of what in the topology literature is known as the 'doubling construction'. Let $\Phi: \mathcal{U}(q) \rightarrow \mathcal{U}(q)$ be defined as follows:

$$
\Phi M=\operatorname{Im}\left\{S_{q}(M) \hookrightarrow M^{\otimes q} \rightarrow S^{q}(M)\right\} .
$$

Then there are natural projections $\pi_{M}: S_{q j}(M) \rightarrow S_{j}(\Phi M)$, natural inclusions $i_{M}: S^{j}(\Phi M) \rightarrow S^{q j}(M)$, and natural isomorphisms of vector spaces

$$
(\Phi M)_{n} \simeq \begin{cases}M_{n / q} & \text { if } q \text { divides } n \\ 0 & \text { otherwise }\end{cases}
$$

Using this isomorphism, a natural map $\lambda_{M}: \Phi M \rightarrow M$ can be defined by $\lambda_{M}(x)=\Phi(x)$. Then $\lambda_{M}$ is $\mathcal{A}(q)$-linear, and is monic if $M$ is nilclosed.

\footnotetext{
${ }^{5}$ Note that for $K \in \mathcal{K}(q), K$ is nilpotent in our sense if and only if it is a nilpotent algebra in the usual algebraic sense.
} 


\section{Proof of Theorem 1.3}

The first cases of Theorem 1.3 follow from just the Kunneth formula of the introduction, and the fact that $r$ is left exact.

To prove case (1), we start with the observation that there is an exact sequence

$$
0 \longrightarrow S_{n} \longrightarrow T^{n} \stackrel{\prod_{i=1}^{n-1}\left(1-w_{i}\right)}{\longrightarrow} \prod_{i=1}^{n-1} T^{n}
$$

where $w_{i} \in \Sigma_{n}, i=1, \ldots, n-1$, are transpositions generating the $n^{\text {th }}$ symmetric group. Precomposing with $G$ yields

$$
0 \longrightarrow S_{n} \circ G \longrightarrow G^{\otimes n} \stackrel{\prod_{i=1}^{n-1}\left(1-w_{i}\right)}{\longrightarrow} \prod_{i=1}^{n-1} G^{\otimes n} .
$$

Applying $r$ and the Kunneth formula to this yields the exact sequence in $\mathcal{U}(q)$,

$$
0 \longrightarrow M_{S_{n} \circ G} \longrightarrow M_{G}^{\otimes n} \stackrel{\prod_{i=1}^{n-1}\left(1-w_{i}\right)}{\longrightarrow} \prod_{i=1}^{n-1} M_{G}^{\otimes n},
$$

which shows that $M_{S_{n} \circ G} \simeq S_{n}\left(M_{G}\right)$.

If char $\mathbf{F}_{q}$ is not 2, case (2) follows from a similar proof, with $\prod_{i=1}^{n-1}\left(1-w_{i}\right)$ replaced by $\prod_{i=1}^{n-1}\left(1+w_{i}\right)$. If char $\mathbf{F}_{q}=2, \Lambda^{n}=\bar{S}^{n}$, so is included in case (3).

To prove case (3), we start by recalling [K:II, Example 7.6] that $\bar{S}^{n}$ is the image of the 'norm' $N: S^{n} \rightarrow S_{n}$, and thus is self dual (and simple). One sees also that case (3) is a special subcase of case (4), but if $q=p$ (i.e. is prime) one can continue as follows.

There is an exact sequence in $\mathcal{F}(p)$,

$$
\bigoplus_{j \geq 1} S^{j} \otimes S^{n-p j} \longrightarrow S^{n} \longrightarrow \bar{S}^{n} \longrightarrow 0
$$

where $S^{j} \otimes S^{n-p j} \longrightarrow S^{n}$ is the composite

$$
S^{j} \otimes S^{n-p j} \stackrel{\Phi \otimes 1}{\longrightarrow} S^{p j} \otimes S^{n-p j} \longrightarrow S^{n}
$$

Dualizing yields the exact sequence in $\mathcal{F}(p)$,

$$
0 \longrightarrow \bar{S}^{n} \longrightarrow S_{n} \longrightarrow \prod_{j \geq 1} S_{j} \otimes S_{n-p j}
$$

Precomposing with $G$, and then applying $r$, yields an isomorphism in $\mathcal{U}(p)$

$$
0 \longrightarrow M_{\bar{S}^{n} \circ G} \longrightarrow S_{n}\left(M_{G}\right) \longrightarrow \prod_{j \geq 1} S_{j}\left(M_{G}\right) \otimes S_{n-p j}\left(M_{G}\right),
$$

where $S_{n}\left(M_{G}\right) \longrightarrow S_{j}\left(M_{G}\right) \otimes S_{n-p j}\left(M_{G}\right)$ is the composition

$$
S_{n}\left(M_{G}\right) \longrightarrow S_{p j}\left(M_{G}\right) \otimes S_{n-p j}\left(M_{G}\right) \stackrel{r(\Phi) \otimes 1}{\longrightarrow} S_{j}\left(M_{G}\right) \otimes S_{n-p j}\left(M_{G}\right) .
$$

(Here, by abuse of notation, we denote the dual of $\Phi: S^{j} \rightarrow S^{j p}$ by ' $\Phi$ '.)

Meanwhile, using (3.1), one can deduce an exact sequence in $\mathcal{U}(p)$,

$$
0 \longrightarrow \bar{S}^{n}(M) \longrightarrow S_{n}(M) \longrightarrow \prod_{j \geq 1} S_{j}(\Phi M) \otimes S_{n-p j}(M),
$$


where $S_{n}(M) \longrightarrow S_{j}(\Phi M) \otimes S_{n-p j}(M)$ is the composition

$$
S_{n}(M) \longrightarrow S_{p j}(M) \otimes S_{n-p j}(M) \stackrel{\pi_{M} \otimes 1}{\longrightarrow} S_{j}(\Phi M) \otimes S_{n-p j}(M) .
$$

Equation (3.3) can be then compared with (3.2), yielding case (3) in the $q=p$ subcase, using the following addendum to case (1).

Proposition 3.1. Under the isomorphism $S_{n}\left(M_{G}\right) \simeq M_{S_{n} \circ G}$, the map $r(\Phi)$ : $M_{S_{q j} \circ G} \longrightarrow M_{S_{j} \circ G}$ corresponds to the composite $S_{q j}\left(M_{G}\right) \stackrel{\pi_{M}}{\longrightarrow} S_{j}\left(\Phi M_{G}\right) \stackrel{S_{j}(\lambda)}{\longrightarrow}$ $S_{j}\left(M_{G}\right)$.

To prove this, one can immediately reduce to the case $j=1$, which can be easily checked directly. (Compare with [K1, Proposition 6.7 and its proof].)

We end this section with the more subtle proof of case (4) of Theorem 1.3.

Restated, this says the following.

Proposition 3.2. For any $c \in \mathbf{F}_{q}\left[\Sigma_{n}\right]$, and locally finite $G \in \mathcal{F}(q)$, the natural inclusion

$$
c \operatorname{Hom}_{\mathcal{F}(q)}\left(S_{*}, G^{\otimes n}\right) \subseteq \operatorname{Hom}_{\mathcal{F}(q)}\left(S_{*}, c G^{\otimes n}\right)
$$

is an isomorphism.

Via duality (and the fact that $r$ commutes with filtered direct limits), this is equivalent to

Proposition 3.3. For any $c \in \mathbf{F}_{q}\left[\Sigma_{n}\right]$, and finite $G \in \mathcal{F}(q)$, the natural inclusion

$$
c \operatorname{Hom}_{\mathcal{F}(q)}\left(G^{\otimes n}, S^{*}\right) \subseteq \operatorname{Hom}_{\mathcal{F}(q)}\left(G^{\otimes n} c, S^{*}\right)
$$

is an isomorphism.

This we now prove, following the line of argument in [KK, §3]. Noting that $\operatorname{Hom}_{\mathcal{F}(q)}\left(G^{\otimes n} c, S^{*}\right) \subseteq \operatorname{Hom}_{\mathcal{F}(q)}\left(G^{\otimes n}, S^{*}\right)=M_{D G}^{\otimes n}$, Proposition 3.3 will follow from the next two lemmas.

Lemma 3.4. In the situation of Proposition 3.3, for all $x \in \operatorname{Hom}_{\mathcal{F}(q)}\left(G^{\otimes n} c, S^{j}\right)$, there exists a $k$ so that $\Phi^{k}(x) \in c \operatorname{Hom}_{\mathcal{F}(q)}\left(G^{\otimes n}, S^{q^{k} j}\right)$.

Lemma 3.5. Given $c \in \mathbf{F}_{q}\left[\Sigma_{n}\right]$ and $M \in \mathcal{U}_{\Phi}(q)$ such that $\Phi: M \rightarrow M$ is monic, if $\Phi^{k}(x) \in c M^{\otimes n}$, then $x \in c M^{\otimes n}$.

Proof of Lemma 3.4. The Vanishing Theorem, Theorem 2.4, asserts the injectivity of $\Phi^{-1} S^{j}$. It follows that, given $x: G^{\otimes n} c \rightarrow S^{j}$, there is an extension



The image of $y$ will be contained in $S^{q^{k} j}$ for $k$ large enough. But this means that $\Phi^{k}(x) \in c \operatorname{Hom}_{\mathcal{F}(q)}\left(G^{\otimes n}, S^{q^{k} j}\right)$, as needed. 
Proof of Lemma 3.5. The lemma follows from the claim, to be proven below, that

$$
0 \rightarrow \Phi^{k}\left(M^{\otimes n}\right) \rightarrow M^{\otimes n} \rightarrow M^{\otimes n} / \Phi^{k}\left(M^{\otimes n}\right) \rightarrow 0
$$

splits as a short exact sequence of $\mathbf{F}_{q}\left[\Sigma_{n}\right]$-modules. (Here $\Phi^{k}\left(M^{\otimes n}\right)$ means $\operatorname{Im}\left\{\Phi^{k}: M^{\otimes n} \rightarrow M^{\otimes n}\right\}$.) For then, if $\Phi^{k}(x) \in c M^{\otimes n}$, there exists a $y \in M^{\otimes n}$ such that $\Phi^{k}(x)=c \Phi^{k}(y)=\Phi^{k}(c y)$, and we conclude that $x=c y$.

To see that the claim holds, first note that

$$
\Phi^{k}\left(M^{\otimes n}\right) \simeq\left(\Phi^{k}(M)\right)^{\otimes n},
$$

and then that, for any two (graded) vector spaces $V, W$, there is an isomorphism of $\mathbf{F}_{q}\left[\Sigma_{n}\right]$-modules

$$
(V \oplus W)^{\otimes n} \simeq \bigoplus_{s+t=n} I n d_{\Sigma_{s} \times \Sigma_{t}}^{\Sigma_{n}}\left(V^{\otimes s} \otimes W^{\otimes t}\right)
$$

so that, in particular, $V^{\otimes n} \subseteq(V \oplus W)^{\otimes n}$ splits as $\mathbf{F}_{q}\left[\Sigma_{n}\right]$-modules.

\section{Proofs of Theorems 1.6 And 1.9}

Proof of Theorem 1.6. We begin by explaining why $M_{J_{W} \circ G}$ is naturally an object in $\mathcal{K}(q)$, a.k.a. an unstable $\mathcal{A}(q)$-algebra. The point is that, since $J_{W}(G(V))$ is the ring of functions $\mathbf{F}_{q}^{\operatorname{Hom}(G(V), W)}, J_{W} \circ G$ takes values in $q$-Boolean algebras: commutative $\mathbf{F}_{q}$-algebras $B$ satisfying $x^{q}=x$, for all $x \in B$. Theorem 6.9 of [K1] then says that $r$ takes $q$-Boolean algebra valued functors to unstable $\mathcal{A}(q)$ algebras (and $l$ does the opposite).

We now show that $U\left(M_{G} \otimes W^{*}\right) \simeq M_{J_{W} \circ G}$, in $\mathcal{K}(q)$, if $q$ is prime, by verifying that $U$ satisfies the two conditions of Theorem 2.6.

Recall that $U(M)=S^{*}(M) /\left(\Phi(x)-x^{q}\right)$. Filtering $U(M)$ by polynomial degree shows that $U(M)$ has an associated graded object isomorphic in $\mathcal{U}(q)$ to $S^{*}(M) /\left(x^{q}\right)$. If $q$ is prime, this identifies with $\bar{S}^{*}(M)$, which is nilclosed if $M$ is nilclosed, by case (3) of Theorem 1.3. Thus $U(M)$ is nilclosed if $M$ is, verifying the first condition in Theorem 2.6.

By construction, $U(M)$ is the coequalizer in the category of commutative, unital algebras in $\mathcal{U}(q)$ :

$$
S^{*}(\Phi M) \underset{i_{M}}{\longrightarrow} S^{*}(M) \longrightarrow U(M) \rightarrow 0 .
$$

Since $l$ is exact and preserves tensor products, we deduce that $l\left(U\left(M \otimes W^{*}\right)\right)$ is the coequalizer in the category of commutative algebras in $\mathcal{F}(q)$ :

$$
S^{*}\left(l(\Phi M) \otimes W^{*}\right) \underset{l\left(i_{M}\right)}{\longrightarrow} S^{*}\left(l(M) \otimes W^{*}\right) \longrightarrow l\left(U\left(M \otimes W^{*}\right)\right) \rightarrow 0 .
$$

By Proposition 6.7 of [K1], $l\left(\lambda_{M}\right): l(\Phi M) \rightarrow l(M)$ can be identified with $1: l(M) \rightarrow l(M)$, and, under this identification, $l\left(i_{M}\right): S^{j}(l(\Phi M)) \rightarrow S^{q j}(M)$ 
corresponds to $\Phi: S^{j}(l(M)) \rightarrow S^{q j}(l(M))$. Thus $l\left(U\left(M \otimes W^{*}\right)\right)$ is the coequalizer in the category of commutative algebras in $\mathcal{F}(q)$ :

$$
S^{*}\left(l(M) \otimes W^{*}\right) \underset{\Phi}{\longrightarrow} S^{*}\left(l(M) \otimes W^{*}\right) \longrightarrow l\left(U\left(M \otimes W^{*}\right)\right) \rightarrow 0 .
$$

The next lemma then allows us to conclude that $l\left(U\left(M \otimes W^{*}\right)\right) \simeq J_{W} \circ l(M)$, thus verifying the second condition in Theorem 2.6, and finishing the proof of Theorem 1.6.

Lemma 4.1. $J_{W}(V)$ is naturally the coequalizer in the category of $\mathbf{F}_{q}$-algebras:

$$
S^{*}\left(V \otimes W^{*}\right) \underset{\Phi}{\longrightarrow} S^{*}\left(V \otimes W^{*}\right) \longrightarrow J_{W}(V) \rightarrow 0 .
$$

This is just saying that $J_{W}(V)=S^{*}\left(V \otimes W^{*}\right) /\left(x^{q}-x\right)$, an elementary, but useful, observation made in [K:I, Lemma 4.12].

Remark 4.2. If $q$ is not prime, $U(M)$ need not be nilclosed if $M$ is. For a simple example when $q=4$, let $M=M_{I_{\xi}}$, which has a generator in degrees $2^{2 k+1}, k \geq 0$. Thus $U(M)$ is concentrated in even degrees.

We claim that the nilclosure of $U(M)$ has a nonzero class in degree 1 . Our argument above shows that $l(U(M))=\left(J_{\mathbf{F}_{4}}\right)_{\xi}$, so we are asserting that there is a nonzero map $I \rightarrow\left(J_{\mathbf{F}_{4}}\right)_{\xi}$, or equivalently, a nonzero map $I_{\xi} \rightarrow J_{\mathbf{F}_{4}}$. But $I_{\xi} \subset S^{2}$, and $S^{2} \subset J_{\mathbf{F}_{4}}$, so this is obvious.

Proof of Theorem 1.9. This is an argument similar to the proof of Theorem 1.6 above.

That $M_{S^{*} \circ G}$ is an object in $\mathcal{K}^{2}(q)$, when $G=I$, was shown in [K:III, §5]. The same argument shows this is true for arbitrary $G$.

Now consider the natural map

$M_{G} \otimes M_{I}=\operatorname{Hom}_{\mathcal{F}(q)}\left(S_{*}, G\right) \otimes \operatorname{Hom}_{\mathcal{F}(q)}\left(I, S^{*}\right) \rightarrow \operatorname{Hom}_{\mathcal{F}(q)}\left(S_{*}, S^{*} \circ G\right)=M_{S^{*} \circ G}$

that sends $\left(S_{m} \stackrel{x}{\rightarrow} G\right) \otimes\left(I \stackrel{\Phi^{k}}{\longrightarrow} S^{q^{k}}\right)$ to the composite $S_{m} \stackrel{x}{\longrightarrow} G \stackrel{\Phi^{k} \circ 1}{\longrightarrow} S^{q^{k}} \circ G$. This is a map in $\mathcal{U}^{2}(q)$, and thus extends uniquely to a map in $\mathcal{K}^{2}(q)$,

$$
U_{2}\left(M_{G} \otimes M_{I}\right) \longrightarrow M_{S^{*} \circ G} .
$$

We show that this is an isomorphism, when $q$ is prime, by checking the two conditions in Theorem 2.6.

Firstly, to see that $U_{2}\left(M \otimes M_{I}\right)$ is nilclosed (with respect to the first grading) if $M$ is nilclosed, one can filter $U_{2}\left(M \otimes M_{I}\right)=S^{*}\left(M \otimes M_{I}\right) /\left(\Phi(x)-x^{q}\right)$, obtaining an associated graded object isomorphic in $\mathcal{U}^{2}(q)$ to $\bar{S}^{*}\left(M \otimes M_{I}\right)$ (since $q$ is prime). Ignoring the second grading, Theorem 1.3 shows this is nilclosed if $M$ is.

By construction, $U_{2}\left(M \otimes M_{I}\right)$ is the coequalizer in the category of commutative, unital algebras in $\mathcal{U}^{2}(q)$ :

$$
S^{*}\left(\Phi M \otimes \Phi M_{I}\right) \stackrel{\lambda_{M} \otimes \lambda_{M_{I}}}{\longrightarrow} \underset{i_{M}}{\longrightarrow} S_{M_{I}}\left(M \otimes M_{I}\right) \longrightarrow U_{2}\left(M \otimes M_{I}\right) \rightarrow 0 .
$$


Since $l$ is exact and preserves tensor products, we deduce that $l\left(U_{2}\left(M \otimes M_{I}\right)\right)$ is the coequalizer in the category of graded commutative algebras in $\mathcal{F}(q)$ :

$$
S^{*}\left(l(M) \otimes \Phi M_{I}\right) \underset{i_{M_{I}}}{\longrightarrow} S^{*}\left(l(M) \otimes M_{I}\right) \longrightarrow l\left(U_{2}\left(M \otimes M_{I}\right)\right) \rightarrow 0 .
$$

Thus, for any vector space $V, l\left(U_{2}\left(M \otimes M_{I}\right)\right)(V)$ is naturally isomorphic to $U\left(l(M)(V) \otimes M_{I}\right)$ (where now we are using the $\mathcal{U}(q)$ stucture on $\left.M_{I}\right)$.

The next lemma then allows us to conclude that $l\left(U_{2}\left(M \otimes M_{I}\right)\right) \simeq S^{*} \circ l(M)$, thus verifying the second condition in Theorem 2.6, and finishing the proof of Theorem 1.9.

Lemma 4.3. For any vector space $W, U\left(W \otimes M_{I}\right)$ is naturally isomorphic as an algebra to $S^{*}(W)$.

This is easily checked (and well known).

Remark 4.4. Analogous to the situation in Remark 4.2, if $q$ is not prime, $U_{2}\left(M \otimes M_{I}\right)$ need not be nilclosed if $M$ is. With $q=4$ and $M=M_{I_{\xi}}$, $U_{2}\left(M \otimes M_{I}\right)$ is concentrated in bidegrees $(m, n)$ with $m$ even. However its nilclosure has a nonzero class in bidegree $(1,2)$. This is equivalent to asserting that there is a nonzero map $I \rightarrow S_{\xi}^{2}$, or equivalently, a nonzero map $I_{\xi} \rightarrow S^{2}$. As noted before, $I_{\xi} \subset S^{2}$, and so this is obvious.

\section{Some Comments on the Frobenius twist}

Let $q=p^{s}$, with $p$ prime. Let $\mathcal{A}(q)^{*}$ be the Hopf algebra dual to $\mathcal{A}(q)$. As noted in [K:III, $\S 8]$, the $q$ analogue of Milnor's calculation [Mn] holds: as graded algebras

$$
\mathcal{A}(q)^{*} \simeq \mathbf{F}_{q}\left[\xi_{1}, \xi_{2}, \ldots\right]
$$

where the degree of $\xi_{i}$ is $q^{i}-1$. Using the monomial basis for $\mathcal{A}(q)^{*}$, let $Q^{i} \in \mathcal{A}(q)$ be dual to $\xi_{i} \in \mathcal{A}(q)^{*}$. By their construction, one sees that, on $S^{*}\left(\mathbf{F}_{q}\right)=\mathbf{F}_{q}[x]$, where $x$ has degree $1, Q^{i} x=x^{q^{i}}$, and also that $Q^{i}$ acts as a derivation on $\mathcal{A}(q)$ algebras. Following the discussion in [AW, $\S 5]$, we thus conclude that if $V_{l}$ has basis $x_{1}, \ldots, x_{l}$, then

$$
Q^{i}=\sum_{j=1}^{l} a_{i j} \partial / \partial x_{j}: S^{*}\left(V_{l}\right) \rightarrow S^{*}\left(V_{l}\right)
$$

where $a_{i j}=\left(x_{j}\right)^{q^{i}}$.

The proof of Lemma 5.9 of [AW] works with $p$ replaced by $q$, to show that the matrix $\left(a_{i j}\right), 1 \leq i, j \leq l$, is nonsingular. It follows that, for any $\mathbf{F}_{q}$-vector space $V$,

$$
\bigcap_{i \geq 1} \operatorname{Ker}\left\{Q^{i}: S^{*}(V) \rightarrow S^{*}(V)\right\}
$$

is the collection of all polynomials with all partial derivatives vanishing. But this is precisely $S^{*}(V)_{\xi}$, with grading multiplied by $p$, embedded in $S^{*}(V)$ as the $p^{\text {th }}$ powers. We have proved 
Lemma 5.1. There is an exact sequence in $\mathcal{F}(q)$ :

$$
0 \longrightarrow S_{\xi}^{*} \longrightarrow S^{*} \stackrel{\prod_{i} Q^{i}}{\longrightarrow} S^{*}
$$

There is, of course, a dual sequence involving $S_{*}$.

This suggests the definition of a functor $U_{\xi}: \mathcal{U}(q) \rightarrow \mathcal{U}(q)$. As graded vector spaces,

$$
U_{\xi}(M)_{n}=\bigcap_{i \geq 1} \operatorname{Ker}\left\{Q^{i}: M_{p n} \rightarrow M_{p n+q^{i}-1}\right\} .
$$

To see that this has a natural unstable $\mathcal{A}(q)$-module structure, we again follow the lead of $[\mathrm{AW}]$.

The $p^{\text {th }}$ power map $\mathcal{A}(q)^{*} \rightarrow \mathcal{A}(q)^{*}$ is a monomorphism of Hopf algebras. Dual to this is an epic map $\phi: \mathcal{A}(q) \rightarrow \mathcal{A}(q)$, which divides degree by $p$ in degrees divisible by $p$, and is 0 otherwise.

Lemma 5.2. $\operatorname{Ker} \phi=\mathcal{A}(q)\left\{Q^{1}, Q^{2}, \ldots\right\}$, which is thus a two sided ideal.

Adams and Wilkerson's proof of the $q=p$ case, Lemma 2.5 of [AW], generalizes immediately.

The $\mathcal{A}(q)$-module structure on $U_{\xi}(M)$ is then defined to correspond to the evident action of $\mathcal{A}(q) / \mathcal{A}(q)\left\{Q^{1}, Q^{2}, \ldots\right\}$ on $\bigcap_{i>1} \operatorname{Ker}\left\{Q^{i}: M \rightarrow M\right\}$.

Since $M_{G_{\xi^{-1}}}=\operatorname{Hom}_{\mathcal{F}(q)}\left(S_{*}, G_{\xi^{-1}}\right) \simeq \operatorname{Hom}_{\mathcal{F}(q)}\left(\left(S_{*}\right)_{\xi}, G\right)$, Lemma 5.1 immediately implies Theorem 1.12: $U_{\xi}\left(M_{G}\right) \simeq M_{G_{\xi^{-1}}}$.

Remark 5.3. One can imagine extensions of some of our results, where one inputs knowledge of $M_{G_{\xi^{i}}}$, for $0 \leq i \leq s-1$, to compute $M_{F \circ G_{\xi^{i}}}$, for all $i$. By the generic

Steinberg Tensor Product Theorem [K:II, Theorem 5.23], case (4) of Theorem 1.3 here gives such a result, for any simple functor $F \in \mathcal{F}(q)$.

\section{REFERENCES}

[AW] J. F. Adams and C. W. Wilkerson, Finite H-spaces and algebras over the Steenrod algebra, Ann. Math. 111 (1980), 95-143. MR 81h:55006

[EM] S. Eilenberg and S. Mac Lane, On the groups $H(\pi, n)$, II, Ann. Math. 60 (1954), 49-139. MR 16:392a

[FS] V. Franjou and L. Schwartz Reduced unstable A-modules and the modular representation theory of the symmetric groups, Ann. Sci. École Norm. Sup. 23(1990), 593-624. MR 91k:55019

[G] P. Gabriel, Des catégories abéliennes, Bull. Soc. Math. France 90(1962), 323-348. MR 38:1144

[HLS] H.-W. Henn, J. Lannes, and L. Schwartz The categories of unstable modules and unstable algebras modulo nilpotent objects, Amer. J. Math. 115(1993), 1053-1106.

[KK] P. Krason and N. J. Kuhn, On embedding polynomial functors in symmetric powers, J. Algebra. 163(1993), 281-294. MR 95e:20058

[K:I] N. J. Kuhn, Generic representation theory of the finite general linear groups and the Steenrod algebra: I, Amer. J. Math. 116(1994), 327-360. MR 95c:55022

[K:II] N. J. Kuhn, Generic representation theory of the finite general linear groups and the Steenrod algebra: II, K-Theory J. 8(1994), 395-428. MR 95k:55038

[K:III] N. J. Kuhn, Generic representation theory of the finite general linear groups and the Steenrod algebra: III, K-Theory J. 9(1995), 273-303. MR 97c:55026

[K1] N. J. Kuhn, Generic representation theory and Lannes' T-functor, Adams Memorial Symposium on Algebraic Topology, Vol. 2, London Math. Soc. Lect. Note Series 176(1992), 235-262. MR 94i:55025

[K2] N. J. Kuhn, New cohomological relationships among loopspaces, symmetric products, and Eilenberg Mac Lane spaces, preprint, 1996. 
[L] J.Lannes, Sur les espaces fonctionnels dont la source est le classifiant d'un p-groupe abélien élémentaire, Pub. I.H.E.S. 75(1992), 135-244. MR 93j:55019

[LS] J.Lannes and L.Schwartz, Sur la structure des A-modules instables injectifs, Topology 28(1989), 153-169. MR 90h:55027

[MacD] I. G. MacDonald, Symmetric functions and Hall Polynomials, Oxford Math. Monographs, Oxford University Press, New York, 1979. MR 84g:05003

[Mn] J. Milnor, The Steenrod algebra and its dual, Ann. Math. 67(1958), 150-171. MR 20:6092

[P] N. Popescu, Abelian Categories with Applications to Rings and Modules, Academic Press, London, 1973. MR 49:5130

[SE] N. E. Steenrod and D. P. A. Epstein, Cohomology Operations, Annals of Math Studies 50, Princeton University Press, Princeton, 1962. MR 26:3056

Department of Mathematics, University of Virginia, Charlottesville, Virginia 22903

E-mail address: njk4x@virginia.edu 